\title{
Peltaea obsita (Mart. ex Colla) Krapov. \& Cristóbal nov. comb. (Malvaceae)
}

\author{
por ANTONIO KRAPOVICKAS y CARMEN L.CRISTOBAL'
}

\begin{abstract}
Summary: The new combination Peltaea obsita (Mart. ex Colla) Krapov. \& Cristóbal is proposed after the finding of the holotype of Sida obsita Martius ex Colla (TO).
\end{abstract}

Luigi Colla publicó en 1833 varias especies nuevas de Malvales en el volumen 1 del Herbarium Pedemontanum, basadas sobre ejemplares brasileños enviados por Martius. Algunas de las Malváceas fueron estudiadas por Fryxell (1976), quién actualizó la posición y estableció las correspondientes sinonimias de seis de ellas. Sida obsita Martius ex Colla no figura en dicho estudio, seguramente porque en el herbario del Istituto ed Orto Botanico dell'Universitá (TO), Torino, Italia, donde se encuentra la colección Colla, esta especie está caratulada por error como Sida obteica.

En la etiqueta del ejemplar dice: «Sida obsita M, Almeida velha, Brasil, 1827, Martius» con lo cual no quedan dudas sobre su autenticidad. La localidad no figura en los itinerarios de Martius (Urban, 1906). Como Martius solía incluir en su herbario plantas cedidas por otros coleccionistas no sería aventurado suponer que el ejemplar de Sida obsita naya tenido un origen similar. Almeida (ahora Nova Almeida) es una localidad que fue visitada, antes de 1827 , por el Príncipe Wied-Neuwied y Sello en 1815 y por Saint Hilaire en 1817. Según el diario de viaje de Míaximiliano, Príncipe de WiedNeuwiec' (1958: 149-151) Vila Velha o Aldeia Velha está sobre el río Piraqué Açu, a poca distancia al norte de Vila Nova de Almeida, a orillas del río dos Reis Magos (ca. $20^{\circ}$ lat. S).

\footnotetext{
' Instituto de Botánica del Nordeste, C. C. 209, 3400 Corrientes, Rep. Argentina.
}

Según St. Hilaire (1974: 66), los jesuitas habían preferido la posición de Vila Nova en lugar de Aldeia Velha por estar en ella más protegidos los indígenas a su cargo.

Peltaea obsita (Mart. ex Colla) Krapov. \& Cristóbal, nov. comb.

Sida obsita Martius ex Colla, 1833. Herb. Ped. 1: 416, «Brasil:, Almeida, Velua». Holotipo: BRASIL, [Espirito Santo], Almeida Velha, 1827, Martius (TO!).

Peltostegia parviflora Turcz. 1858. Bull. Soc. Imp. Naturalistes Moscou 31: 224. Holotipo: BRASIL, Piahuy, Goiáz, \&c. 1837-1841, Gardner s.n. (KW).

Pavonia sessiliflora H. B. K. var. acutifolin Gürke. 1892. Mart. Fl. bras. 12(3): 493. Lectotipo (aquí elegido): Rio de Janeiro, Glaziou 7879 (P!). Isolectotipo: C (foto F 21601!).

Peltaea acutifolia (Gürke) Krapov. \& Cristóbal. 1965. Kurtziana 2: 165-168, fig. 4, A \& B.

Peltaea parviflora (Turcz.) Fryxell \& Krapov. 1990. Contr. Univ. Mich. Herb. 17: 179.

\section{Bibliografía}

COLLA, Luigi A. 1833-1837. Herbarium Pedemontanum. Torino. 8 vols.

FRYXELL, P.A. 1976. On some brazilian Malvaceae of Martius, published by Colla in 1833. Taxon 25(5/6): 589-593.

SAINT HILAIRE, A. 1974. Viagem ao Espirito Santo e Rio Doce. Belo Horizonte. 121 págs.

URBAN, I. 1906. Vitne itineraque collectorum botnnicorum, Mart. Fl. bras. 1(1): 1-154.

WIED-NEUWIED, MAXIMILIANO PRINCIPE DO, 1958. Viagem ao Brasil nos anos de 1815 a 1817. São Paulo. 536 págs. 